\title{
A Regularity Criterion for the 3D Incompressible Magnetohydrodynamics Equations in the Multiplier Spaces
}

\author{
Chunhong Tian \\ Nanhang Jincheng College, Nanjing 211156, China \\ Correspondence should be addressed to Chunhong Tian; chunhongtian@yeah.net
}

Received 5 March 2017; Accepted 18 June 2017; Published 27 August 2017

Academic Editor: Xinguang Zhang

Copyright ( 2017 Chunhong Tian. This is an open access article distributed under the Creative Commons Attribution License, which permits unrestricted use, distribution, and reproduction in any medium, provided the original work is properly cited.

We are concerned with the regularity criterion for weak solutions to the $3 \mathrm{D}$ incompressible MHD equations in this paper. We show that if some partial derivatives of the velocity components and magnetic components belong to the multiplier spaces, then the solution actually is smooth on $(0, T)$.

\section{Introduction}

We consider the 3D incompressible magnetohydrodynamics (MHD) equations.

(MHD)

$$
\begin{aligned}
\frac{\partial u}{\partial t}-v \Delta u+u \cdot \nabla u & =-\nabla p-\frac{1}{2} \nabla b^{2}+b \cdot \nabla b \\
\frac{\partial b}{\partial t}-\eta \Delta b+u \cdot \nabla b & =b \cdot \nabla u \\
\nabla \cdot u & =\nabla \cdot b=0 \\
u(0, x) & =u_{0}(x) \\
b(0, x) & =b_{0}(x) .
\end{aligned}
$$

Here $u, b$ describe the flow velocity vector and the magnetic field vector, respectively, $p$ is a scalar pressure, $v>0$ is the kinematic viscosity, and $\eta>0$ is the magnetic diffusivity, while $u_{0}$ and $b_{0}$ are the given initial velocity and initial magnetic field, respectively, with $\nabla \cdot u_{0}=\nabla \cdot b_{0}=0$. If $v=\eta=0$, (1) is called the ideal MHD equations.

Employing the standard energy method, it is easy to prove that, for given initial data $\left(u_{0}, b_{0}\right) \in H^{s}\left(\mathbb{R}^{3}\right)$ with $s>1 / 2$, there exist a positive time $T=T\left(\left\|\left(u_{0}, b_{0}\right)\right\|_{H^{s}}\right)$ and a unique smooth solution $(u(t, x), b(t, x))$ on $[0, T)$ to the MHD equations satisfying

$$
\begin{aligned}
(u, b) \in & C\left([0, T) ; H^{s}\right) \cap C^{1}\left((0, T) ; H^{s}\right) \\
& \cap C\left((0, T) ; H^{s+2}\right) .
\end{aligned}
$$

Whether smooth solutions of (1) on $[0, T)$ will lead to a singularity at $t=T$ is an outstanding open problem; see Sermange and Temam [1].

However, the solution regularity can be derived when certain growth conditions are satisfied. For regularity of the weak solutions to the 3D MHD equations (1), some numerical experiments $[2,3]$ seem to indicate that the velocity field plays a more important role than the magnetic field in the regularity theory of solutions to the MHD equations. Recently, inspired by Constantin and Fefferman initial work [4] where the regularity condition of the direction of vorticity was used to describe the regularity criterion to the NavierStokes equations, He and Xin [5] extended it to the MHD equations and obtained some integrability condition of the magnitude of the only velocity $u$ alone; that is,

$$
\int_{0}^{T}\|u(t)\|_{p}^{q} d t<\infty, \quad \frac{2}{q}+\frac{3}{p} \leq 1,3<p \leq \infty .
$$

Later, Zhou and Gala [6] extended it to the multiplier spaces. Other important studies such as the regularity criteria can be found in [7-9] and references therein. 
Recently, some regularity criteria in terms of partial velocity components and magnetic components or partial derivative of the velocity components and magnetic components were established [10-12]. However, the spaces used are not scaling invariant (in other words, not of Serrin's type). Many researchers were devoted to studying it along this direction. In 2010, Ji and Lee [13] obtained the following regularity:

$$
\begin{aligned}
& \tilde{u} \in L^{p}\left([0, T) ; L^{q}\left(\mathbb{R}^{3}\right)\right), \\
& \tilde{b} \in L^{l}\left([0, T) ; L^{m}\left(\mathbb{R}^{3}\right)\right), \\
& \frac{2}{p}+\frac{3}{q} \leq 1, \frac{2}{l}+\frac{3}{m} \leq 1,3<q, m \leq \infty .
\end{aligned}
$$

In 2012, Ni et al. [14] got some new regularity as follows:

$$
\begin{aligned}
& \widetilde{\nabla} u \in L^{p}\left([0, T) ; L^{q}\left(\mathbb{R}^{3}\right)\right), \\
& \widetilde{\nabla} b \in L^{l}\left([0, T) ; L^{m}\left(\mathbb{R}^{3}\right)\right), \\
& \frac{2}{p}+\frac{3}{q} \leq 2, \frac{2}{l}+\frac{3}{m} \leq 2, \frac{3}{2}<q, m \leq \infty .
\end{aligned}
$$

Our purpose in this paper is to obtain a new regularity criterion of weak solution for the 3D MHD equations in a sense of scaling invariant by employing a different decomposition for nonlinear terms.

Definition 1 (see [1]). A weak solution pair $(u, b)$ of the 3D MHD equations (1) is regular in $[0, T) \times H^{1}\left(\mathbb{R}^{3}\right)$ provided that $(u, b) \in L^{\infty}\left(0, T ; H^{1}\left(\mathbb{R}^{3}\right)\right)$.

Notation 1. Throughout the paper, we use the following notations for the simplicity. The planar components of $(u, b)$ will be denoted by $\widetilde{u}=\left(u_{1}, u_{2}\right)$ and $\widetilde{b}=\left(b_{1}, b_{2}\right)$ and $\widetilde{\nabla}$ will be used for $\tilde{\nabla}=\left(\partial_{1}, \partial_{2}\right)$.

Now we state our result as follows.

Theorem 2. Let $\left(u_{0}, b_{0}\right) \in H^{1}$ with $\nabla \cdot u_{0}=\nabla \cdot b_{0}=0$. If the weak solutions $(u, b)$ to (1) satisfy the following integrability conditions:

$$
\widetilde{\nabla} \widetilde{u}, \widetilde{\nabla} \widetilde{b} \in L^{2 /(2-r)}\left([0, T) ; \dot{X}_{r}\left(\mathbb{R}^{3}\right)\right), \quad \text { with } r \in[0,1),
$$

then the solution $(u, b)$ remains smooth on $[0, T]$.

Remark 3. Since the components of $\widetilde{\nabla} \widetilde{u}$ and $\widetilde{\nabla} \widetilde{b}$ without $u_{3}$ and $b_{3}$ are less than those of $\widetilde{\nabla} u$ and $\widetilde{\nabla} b$, therefore, our result improves that in [14].

\section{Remark 4. Since}

$$
L^{3 / r}\left(\mathbb{R}^{3}\right) \hookrightarrow L^{3 / r, \infty}\left(\mathbb{R}^{3}\right) \hookrightarrow \dot{X}_{r}\left(\mathbb{R}^{3}\right)
$$

as $0 \leq r \leq 3 / 2$, where $L^{p, \infty}$ denotes the weak $L^{p}$-space, therefore, our result extends that in [14].

\section{Preliminaries}

First, we recall the definition and some properties of the multiplier space $\dot{X}_{r}$ introduced recently by Lemarié-Rieusset [15] (see also [16]). The space $\dot{X}_{r}$ of pointwise multipliers which map $L^{2}$ into $\dot{H}^{-r}$ is defined in the following way.

Definition 5. For $0 \leq r<3 / 2$, we define the homogeneous space $\dot{X}_{r}$ by

$$
\dot{X}_{r}=\left\{f \in L_{\mathrm{loc}}^{2}: \forall g \in \dot{H}^{r}, f g \in L^{2}\right\},
$$

where we denote by $\dot{H}^{r}\left(\mathbb{R}^{d}\right)$ the completion of the space $\mathscr{D}\left(\mathbb{R}^{d}\right)$ with respect to the norm $\|u\|_{\dot{H}^{r}}=\left\|(-\Delta)^{1 / 2} u\right\|_{L^{2}}=$ $\left\||\xi|^{r} \widehat{u}(\xi)\right\|_{L^{2}}$, where $\widehat{u}(\xi)$ denotes the Fourier transform of $u$.

The norm of $\dot{X}_{r}$ is given by the operator norm of pointwise multiplication

$$
\|f\|_{\dot{X}_{r}}=\sup _{\|g\|_{\dot{H}^{r}} \leq 1}\|f g\|_{L^{2}}<\infty .
$$

It is easy to check that

$$
\begin{aligned}
\|f(\lambda \cdot)\|_{\dot{X}_{r}} & \leq \frac{1}{\lambda^{r}}\|f\|_{\dot{X}_{r}}, \quad \lambda>0, \\
\left\|f\left(\cdot+x_{0}\right)\right\|_{\dot{X}_{r}} & =\|f\|_{\dot{X}_{r}} .
\end{aligned}
$$

Hence, for any function $f(x, t)$ defined for both spatial and time variables,

$$
\left\|f_{\lambda}\right\|_{L^{2}\left([0, T) ; \dot{X}_{r}\right)}=\|f\|_{L^{2}\left([0, T) ; \dot{X}_{r}\right)},
$$

for any $\lambda>0$, with $f_{\lambda}(x, t)=\lambda f\left(\lambda x, \lambda^{2} t\right)$. Therefore, if $(u, b)$ solves the MHD equations, then so does $\left(u_{\lambda}, b_{\lambda}\right)$. This is the so-called scaling dimension zero property.

Lemma 6. Assume $0 \leq r \leq 1$. Then the following inequality holds:

$$
\|u\|_{\dot{H}^{r}} \leq\|u\|_{L^{2}}^{1-r}\|\nabla u\|_{L^{2}}^{r} .
$$

The proof of this lemma can be easily obtained by Parseval's equality and Hölder's inequality, and we omit it.

\section{Proof of Theorem 2}

By Definition 1, we need to estimate $(u, b) \in L^{\infty}(0, T$; $\left.H^{1}\left(\mathbb{R}^{3}\right)\right)$. Thus, we will divide the proof of Theorem 2 into two steps as follows.

Step 1 ( $L^{2}$-estimates). Multiplying the first equation and the second equation in (1) by $u, b$, respectively, and integrating the resulting equations by parts over $\mathbb{R}^{3}$, we obtain after adding them together from the incompressibility condition $\nabla \cdot u=0$ and $\nabla \cdot b=0$

$$
\begin{aligned}
& \frac{1}{2} \frac{d}{d t} \int\left(|u|^{2}+|b|^{2}\right) d x+\int\left(|\nabla u|^{2}+|\nabla b|^{2}\right) d x \\
& \quad=\langle(-u \cdot \nabla u+b \cdot \nabla b), u\rangle+\langle(-u \cdot \nabla b+b \cdot \nabla u), b\rangle \\
& \quad=0,
\end{aligned}
$$


where $\langle\cdot, \cdot\rangle$ denotes the inner-product in $L^{2}\left(\mathbb{R}^{3}\right)$. Integrating from 0 to $t$ for the above inequality, we have

$$
(u, b) \in L^{\infty}\left([0, T) ; L^{2}\right) \cap L^{2}\left((0, T) ; \dot{H}^{1}\right) .
$$

Step $2\left(\dot{H}^{1}\right.$-estimates). Differentiating the first equation and the second equation of (1) with respect to $x_{k}(1 \leq k \leq 3)$ and multiplying the first and the second equations of (1) by $\partial u / \partial x_{k}=\partial_{k} u$ and $\partial b / \partial x_{k}=\partial_{k} b$, respectively, and then, by integrating by parts over $\mathbb{R}^{3}$, we get

$$
\begin{aligned}
& \frac{1}{2} \frac{d}{d t}\left\|\partial_{k} u\right\|_{L^{2}}^{2}+\left\|\nabla \partial_{k} u\right\|_{L^{2}}^{2} \\
&=-\int \partial_{k}[(u \cdot \nabla) u] \cdot \partial_{k} u d x-\int \partial_{k} \nabla p \cdot \partial_{k} u d x \\
&+\int \partial_{k}[(b \cdot \nabla) b] \cdot \partial_{k} u d x, \\
& \frac{1}{2} \frac{d}{d t}\left\|\partial_{k} b\right\|_{L^{2}}^{2}+\left\|\nabla \partial_{k} b\right\|_{L^{2}}^{2} \\
&=-\int \partial_{k}[(u \cdot \nabla) b] \partial_{k} b d x \\
& \quad+\int \partial_{k}[(b \cdot \nabla) u] \partial_{k} b d x .
\end{aligned}
$$

Noting the incompressibility conditions $\nabla \cdot u=0$ and $\nabla \cdot b=0$, since

$$
\begin{aligned}
& \int \partial_{k}[(u \cdot \nabla) u] \cdot \partial_{k} u d x=\int\left(\partial_{k} u \cdot \nabla\right) u \cdot \partial_{k} u d x, \\
& \int \partial_{k}[(b \cdot \nabla) b] \cdot \partial_{k} u d x \\
& =\int\left(\partial_{k} b \cdot \nabla\right) b \cdot \partial_{k} u d x+\int(b \cdot \nabla) \partial_{k} b \cdot \partial_{k} u d x, \\
& \int \partial_{k} \nabla p \cdot \partial_{k} u d x=0, \\
& \int \partial_{k}[(u \cdot \nabla) b] \partial_{k} b d x=\int\left(\partial_{k} u \cdot \nabla\right) b \partial_{k} b d x, \\
& \int \partial_{k}[(b \cdot \nabla) u] \partial_{k} b d x \\
& =\int\left(\partial_{k} b \cdot \nabla\right) u \partial_{k} b d x+\int(b \cdot \nabla) \partial_{k} u \partial_{k} b d x,
\end{aligned}
$$

then (15) and (16) can be rewritten as

$$
\begin{aligned}
\frac{1}{2} & \frac{d}{d t}\left\|\partial_{k} u\right\|_{L^{2}}^{2}+\left\|\nabla \partial_{k} u\right\|_{L^{2}}^{2} \\
= & -\int\left(\partial_{k} u \cdot \nabla\right) u \cdot \partial_{k} u d x+\int\left(\partial_{k} b \cdot \nabla\right) b \cdot \partial_{k} u d x \\
& +\int(b \cdot \nabla) \partial_{k} b \cdot \partial_{k} u d x, \\
\frac{1}{2} \frac{d}{d t} & \left\|\partial_{k} b\right\|_{L^{2}}^{2}+\left\|\nabla \partial_{k} b\right\|_{L^{2}}^{2}
\end{aligned}
$$

$$
\begin{aligned}
= & -\int\left(\partial_{k} u \cdot \nabla\right) b \partial_{k} b d x+\int\left(\partial_{k} b \cdot \nabla\right) u \partial_{k} b d x \\
& +\int(b \cdot \nabla) \partial_{k} u \partial_{k} b d x .
\end{aligned}
$$

Since $\int(b \cdot \nabla) \partial_{k} u \partial_{k} b d x+\int(b \cdot \nabla) \partial_{k} b \cdot \partial_{k} u d x=0$, adding up (18), we have by summing up over $k(1 \leq k \leq 3)$

$$
\begin{aligned}
\frac{1}{2} \frac{d}{d t}( & \left.\|\nabla u\|_{L^{2}}^{2}+\|\nabla b\|_{L^{2}}^{2}\right)+\left\|\nabla^{2} u\right\|_{L^{2}}^{2}+\left\|\nabla^{2} b\right\|_{L^{2}}^{2} \\
= & -\sum_{k=1}^{3} \int\left(\partial_{k} u \cdot \nabla\right) u \cdot \partial_{k} u d x \\
& +\sum_{k=1}^{3} \int\left(\partial_{k} b \cdot \nabla\right) b \cdot \partial_{k} u d x \\
& -\sum_{k=1}^{3} \int\left(\partial_{k} u \cdot \nabla\right) b \partial_{k} b d x \\
& +\sum_{k=1}^{3} \int\left(\partial_{k} b \cdot \nabla\right) u \partial_{k} b d x \triangleq I_{1}+I_{2}+I_{3}+I_{4} .
\end{aligned}
$$

First, we rewrite $I_{1}$ into seven parts as follows:

$$
\begin{aligned}
I_{1}= & -\sum_{i, j, k=1}^{3} \int \partial_{k} u_{i} \partial_{i} u_{j} \partial_{k} u_{j} d x \\
= & -\sum_{i, j, k=1}^{2} \int \partial_{k} u_{i} \partial_{i} u_{j} \partial_{k} u_{j} d x \\
& -\sum_{j, k=1}^{2} \int \partial_{k} u_{3} \partial_{3} u_{j} \partial_{k} u_{j} d x \\
& -\sum_{i, k=1}^{2} \int \partial_{k} u_{i} \partial_{i} u_{3} \partial_{k} u_{3} d x \\
& -\sum_{i, j=1}^{2} \int \partial_{3} u_{i} \partial_{i} u_{j} \partial_{3} u_{j} d x \\
& -\sum_{k=1}^{3} \int \partial_{k} u_{3} \partial_{3} u_{3} \partial_{k} u_{j} d x-\sum_{i=1}^{2} \int \partial_{3} u_{i} \partial_{i} u_{3} \partial_{3} u_{3} d x \\
& -\sum_{j=1}^{2} \int \partial_{3} u_{3} \partial_{3} u_{j} \partial_{3} u_{j} d x \triangleq \sum_{m=1}^{7} I_{1 m} .
\end{aligned}
$$

According to the definition of $\widetilde{\nabla} \widetilde{u}$, the former four terms of the right-hand side of (20) are bounded by

$$
\left|\sum_{m=1}^{4} I_{1 m}\right| \leq C \int|\widetilde{\nabla} \widetilde{u}\|\nabla u\| \nabla u| d x .
$$


And the latter three terms can be estimated, noting that $\partial_{3} u_{3}=-\partial_{1} u_{1}-\partial_{2} u_{2}$ from the incompressible condition

$$
\begin{aligned}
\left|\sum_{m=5}^{7} I_{1 m}\right| & \leq C \int\left|-\partial_{1} u_{1}-\partial_{2} u_{2}\|\nabla u\| \nabla u\right| d x \\
& \leq C \int|\tilde{\nabla} \tilde{u}\|\nabla u\| \nabla u| d x .
\end{aligned}
$$

Thus, using Hölder's inequality and Young's inequality, we have

$$
\begin{aligned}
I_{1} & \leq C \int|\widetilde{\nabla} \tilde{u}\|\nabla u\| \nabla u| d x \leq C\|\tilde{\nabla} \tilde{u} \nabla u\|_{L^{2}}\|\nabla u\|_{L^{2}} \\
& \leq C\|\widetilde{\nabla} \tilde{u}\|_{\dot{X}^{r}}\|\nabla u\|_{\dot{H}^{r}}\|\nabla u\|_{L^{2}} \\
& \leq C\|\widetilde{\nabla} \tilde{u}\|_{\dot{X}^{r}}\|\nabla u\|_{L^{2}}^{1-r}\left\|\nabla^{2} u\right\|_{L^{2}}^{r}\|\nabla u\|_{L^{2}} \\
& \leq C\|\widetilde{\nabla} \tilde{u}\|_{\dot{X}^{r}}^{2 /(2-r)}\|\nabla u\|_{L^{2}}^{2}+\frac{1}{2}\left\|\nabla^{2} u\right\|_{L^{2}}^{2} .
\end{aligned}
$$

Next, we will bound the term $I_{2}$. We can get, by splitting $I_{2}$ into seven parts,

$$
\begin{aligned}
I_{2}= & \sum_{k=1}^{3} \int\left(\partial_{k} b \cdot \nabla\right) b \cdot \partial_{k} u d x \\
= & \sum_{i, j, k=1}^{3} \int \partial_{k} b_{i} \partial_{i} b_{j} \partial_{k} u_{j} d x \\
= & \sum_{i, j, k=1}^{2} \int \partial_{k} b_{i} \partial_{i} b_{j} \partial_{k} u_{j} d x+\sum_{j, k=1}^{2} \int \partial_{k} b_{3} \partial_{3} b_{j} \partial_{k} u_{j} d x \\
& +\sum_{i, k=1}^{2} \int \partial_{k} b_{i} \partial_{i} b_{3} \partial_{k} u_{3} d x+\sum_{i, j=1}^{2} \int \partial_{3} b_{i} \partial_{i} b_{j} \partial_{3} u_{j} d x \\
& +\sum_{k=1}^{3} \int \partial_{k} b_{3} \partial_{3} b_{3} \partial_{k} u_{j} d x+\sum_{i=1}^{2} \int \partial_{3} b_{i} \partial_{i} b_{3} \partial_{3} u_{3} d x \\
& +\sum_{j=1}^{2} \int \partial_{3} b_{3} \partial_{3} b_{j} \partial_{3} u_{j} d x \triangleq \sum_{m=1}^{7} I_{2 m} .
\end{aligned}
$$

Obviously, the former four terms of the right-hand side of (24) are bounded by

$$
\begin{aligned}
& \left|\sum_{m=1}^{4} I_{2 m}\right| \leq C\left(\int|\widetilde{\nabla} \tilde{u}\|\nabla u\| \nabla b| d x\right. \\
& \left.\quad+\int|\widetilde{\nabla} \tilde{u}\|\nabla b\| \nabla b| d x+\int|\widetilde{\nabla} \widetilde{b}\|\nabla u\| \nabla b| d x\right) .
\end{aligned}
$$

Noting that $\partial_{3} u_{3}=-\partial_{1} u_{1}-\partial_{2} u_{2}$ and $\partial_{3} b_{3}=-\partial_{1} b_{1}-\partial_{2} b_{2}$ from the incompressible conditions, the latter three terms can be estimated as follows:

$$
\begin{aligned}
& \left|\sum_{m=5}^{7} I_{2 m}\right| \leq C\left(\int\left|-\partial_{1} u_{1}-\partial_{2} u_{2}\|\nabla b\| \nabla b\right| d x\right. \\
& \left.\quad+\int\left|-\partial_{1} b_{1}-\partial_{2} b_{2}\|\nabla u\| \nabla b\right| d x\right) \\
& \quad \leq C\left(\int|\widetilde{\nabla} \tilde{u}\|\nabla b\| \nabla b| d x+\int|\widetilde{\nabla} \tilde{b}\|\nabla u\| \nabla b| d x\right) .
\end{aligned}
$$

So, by Hölder's inequality and Young's inequality, we obtain

$$
\begin{aligned}
I_{2} \leq & C\left(\int|\widetilde{\nabla} \tilde{u}\|\nabla u\| \nabla b| d x+\int|\widetilde{\nabla} \widetilde{u}\|\nabla b\| \nabla b| d x\right. \\
& \left.+\int|\widetilde{\nabla} \widetilde{b}\|\nabla u\| \nabla b| d x\right) \leq C\left(\|\widetilde{\nabla} \widetilde{u} \nabla u\|_{L^{2}}\|\nabla b\|_{L^{2}}\right. \\
& \left.+\|\widetilde{\nabla} \tilde{u} \nabla b\|_{L^{2}}\|\nabla b\|_{L^{2}}+\|\widetilde{\nabla} \tilde{b} \nabla b\|_{L^{2}}\|\nabla u\|_{L^{2}}\right) \\
& \leq C\left(\|\widetilde{\nabla} \tilde{u}\|_{\dot{X}^{r}}\|\nabla u\|_{\dot{H}^{r}}\|\nabla b\|_{L^{2}}\right. \\
& \left.+\|\widetilde{\nabla} \tilde{u}\|_{\dot{X}^{r}}\|\nabla b\|_{\dot{H}^{r}}\|\nabla b\|_{L^{2}}+\|\widetilde{\nabla} \tilde{b}\|_{\dot{X}^{r}}\|\nabla u\|_{\dot{H}^{r}}\|\nabla b\|_{L^{2}}\right) \\
& \leq C\left(\|\widetilde{\nabla} \tilde{u}\|_{\dot{X}^{r}}\|\nabla u\|_{L^{2}}^{1-r}\left\|\nabla^{2} u\right\|_{L^{2}}^{r}\|\nabla b\|_{L^{2}}\right. \\
& +\|\widetilde{\nabla} \tilde{u}\|_{\dot{X}^{r}}\|\nabla b\|_{L^{2}}^{1-r}\left\|\nabla^{2} b\right\|_{L^{2}}^{r}\|\nabla b\|_{L^{2}} \\
& \left.+\|\widetilde{\nabla} \tilde{b}\|_{\dot{X}^{r}}\|\nabla u\|_{L^{2}}^{1-r}\left\|\nabla^{2} u\right\|_{L^{2}}^{r}\|\nabla b\|_{L^{2}}\right) \\
& \leq C\left(\|\widetilde{\nabla} \tilde{u}\|_{\dot{X}^{r}}^{2 /(2-r)}+\|\widetilde{\nabla} \tilde{b}\|_{\dot{X}^{r}}^{2 /(2-r)}\right)\left(\|\nabla u\|_{L^{2}}^{2}+\|\nabla b\|_{L^{2}}^{2}\right) \\
& +\frac{1}{2}\left(\left\|\nabla^{2} u\right\|_{L^{2}}^{2}+\left\|\nabla^{2} b\right\|_{L^{2}}^{2}\right) .
\end{aligned}
$$

Similar to the estimate of $I_{2}$, for $I_{3}-I_{4}$, we have

$$
\begin{aligned}
I_{3}+ & I_{4} \\
\leq & C\left(\|\widetilde{\nabla} \widetilde{u}\|_{\dot{X}^{r}}^{2 /(2-r)}+\|\widetilde{\nabla} \widetilde{b}\|_{\dot{X}^{r}}^{2 /(2-r)}\right)\left(\|\nabla u\|_{L^{2}}^{2}+\|\nabla b\|_{L^{2}}^{2}\right) \\
& +\frac{1}{2}\left(\left\|\nabla^{2} u\right\|_{L^{2}}^{2}+\left\|\nabla^{2} b\right\|_{L^{2}}^{2}\right) .
\end{aligned}
$$

Combining the above estimates (23), (27), and (28) into (19), we deduce

$$
\begin{aligned}
\frac{1}{2} \frac{d}{d t}\left(\|\nabla u\|_{L^{2}}^{2}+\|\nabla b\|_{L^{2}}^{2}\right)+\left\|\nabla^{2} u\right\|_{L^{2}}^{2}+\left\|\nabla^{2} b\right\|_{L^{2}}^{2} \\
\leq C\left(\|\widetilde{\nabla} \widetilde{u}\|_{\dot{X}^{r}}^{2 /(2-r)}+\|\widetilde{\nabla} \tilde{b}\|_{\dot{X}^{r}}^{2 /(2-r)}\right)\left(\|\nabla u\|_{L^{2}}^{2}+\|\nabla b\|_{L^{2}}^{2}\right) \\
\quad+\frac{1}{2}\left(\left\|\nabla^{2} u\right\|_{L^{2}}^{2}+\left\|\nabla^{2} b\right\|_{L^{2}}^{2}\right) .
\end{aligned}
$$

Therefore, we have

$$
\begin{aligned}
& \frac{d}{d t}\left(\|\nabla u\|_{L^{2}}^{2}+\|\nabla b\|_{L^{2}}^{2}\right)+\left\|\nabla^{2} u\right\|_{L^{2}}^{2}+\left\|\nabla^{2} b\right\|_{L^{2}}^{2} \\
& \quad \leq C\left(\|\nabla \widetilde{u}\|_{\dot{X}^{r}}^{2 /(2-r)}+\|\widetilde{\nabla} \widetilde{b}\|_{\dot{X}^{r}}^{2 /(2-r)}\right)\left(\|\nabla u\|_{L^{2}}^{2}+\|\nabla b\|_{L^{2}}^{2}\right) .
\end{aligned}
$$


Due to Gronwall's inequality, it follows from (30) that

$$
\begin{aligned}
& \sup _{t \in[0, T]}\left(\|\nabla u\|_{L^{2}}^{2}+\|\nabla b\|_{L^{2}}^{2}\right) \\
& \quad+\int_{0}^{T}\left(\left\|\nabla^{2} u\right\|_{L^{2}}^{2}+\left\|\nabla^{2} b\right\|_{L^{2}}^{2}\right) d t \\
& \leq\left(\left\|\nabla u_{0}\right\|_{L^{2}}^{2}+\left\|\nabla b_{0}\right\|_{L^{2}}^{2}\right) \exp ^{C \int_{0}^{T}\left(\|\widetilde{\nabla} \tilde{u}\|_{\dot{X}^{r}}^{2 /(2-r)}+\|\widetilde{\nabla} \widetilde{b}\|_{\dot{X}^{r}}^{2 /(2-r)}\right) d t .}
\end{aligned}
$$

Thanks to $\widetilde{\nabla} \widetilde{u}, \widetilde{\nabla} \widetilde{b} \in L^{2 /(2-r)}\left([0, T) ; \dot{X}_{r}\left(\mathbb{R}^{3}\right)\right)$, we have

$$
u, b \in L^{\infty}\left([0, T) ; \dot{H}^{1}\right) \cap L^{2}\left([0, T) ; H^{2}\right) .
$$

Combining (14) with (32), we have

$$
u, b \in L^{\infty}\left([0, T) ; H^{1}\right) .
$$

By the standard arguments of continuation of local solutions [1], we can draw the conclusion that the smooth solution $(u, b)$ remains smooth at $t=T$. This completes the proof of Theorem 2 .

\section{Conflicts of Interest}

The author declares that they have no conflicts of interest.

\section{Acknowledgments}

The work was supported by the National Natural Science Foundation of China (11302102) and China Postdoctoral Science Foundation funded project (2014M561893).

\section{References}

[1] M. Sermange and R. Temam, "Some mathematical questions related to the MHD equations," Communications on Pure and Applied Mathematics, vol. 36, no. 5, pp. 635-664, 1983.

[2] A. Hasegawa, "Self-organization processes in continuous media," Advances in Physics, vol. 34, no. 1, pp. 1-42, 1985.

[3] H. Politano, A. Pouquet, and P.-L. Sulem, "Current and vorticity dynamics in three-dimensional magnetohydrodynamic turbulence," Physics of Plasmas, vol. 2, no. 8, pp. 2931-2939, 1995.

[4] P. Constantin and C. Fefferman, "Direction of vorticity and the problem of global regularity for the Navier-Stokes equations," Indiana University Mathematics Journal, vol. 42, no. 3, pp. 775789, 1993.

[5] C. He and Z. Xin, "On the regularity of weak solutions to the magnetohydrodynamic equations," Journal of Differential Equations, vol. 213, no. 2, pp. 235-254, 2005.

[6] Y. Zhou and S. Gala, "Regularity criteria for the solutions to the 3D MHD equations in the multiplier space," Journal of Applied Mathematics and Physics, vol. 61, no. 2, pp. 193-199, 2010.

[7] Q. Chen, C. Miao, and Z. Zhang, "The Beale-Kato-Majda criterion for the 3D magneto-hydrodynamics equations," Communications in Mathematical Physics, vol. 275, no. 3, pp. 861872, 2007.

[8] Q. Chen, C. Miao, and Z. Zhang, "On the regularity criterion of weak solution for the $3 \mathrm{D}$ viscous magneto-hydrodynamics equations," Communications in Mathematical Physics, vol. 284, no. 3, pp. 919-930, 2008.
[9] Q. Chen and C. Miao, "Existence theorem and blow-up criterion of the strong solutions to the two-fluid MHD equation in $\mathbb{R}^{3}$," Journal of Differential Equations, vol. 239, no. 1, pp. 251-271, 2007.

[10] C. Cao and J. Wu, "Two regularity criteria for the 3D MHD equations," Journal of Differential Equations, vol. 248, no. 9, pp. 2263-2274, 2010.

[11] X. Jia and Y. Zhou, "Regularity criteria for the 3D MHD equations involving partial components," Nonlinear Analysis. Real World Applications. An International Multidisciplinary Journal, vol. 13, no. 1, pp. 410-418, 2012.

[12] X. Jia and Y. Zhou, "Regularity criteria for the 3D MHD equations via partial derivatives. II," Kinetic and Related Models, vol. 7, no. 2, pp. 291-304, 2014.

[13] E. Ji and J. Lee, "Some regularity criteria for the 3D incompressible magnetohydrodynamics," Journal of Mathematical Analysis and Applications, vol. 369, no. 1, pp. 317-322, 2010.

[14] L. Ni, Z. Guo, and Y. Zhou, "Some new regularity criteria for the 3D MHD equations," Journal of Mathematical Analysis and Applications, vol. 396, no. 1, pp. 108-118, 2012.

[15] P. G. Lemarié-Rieusset, Recent Developments in the NavierStokes Problem, vol. 431, Chapman \& Hall, Boca Raton, Fla, USA, 2002.

[16] P. G. Lemarié-Rieusset and S. Gala, "Multipliers between Sobolev spaces and fractional differentiation," Journal of Mathematical Analysis and Applications, vol. 322, no. 2, pp. 1030-1054, 2006. 


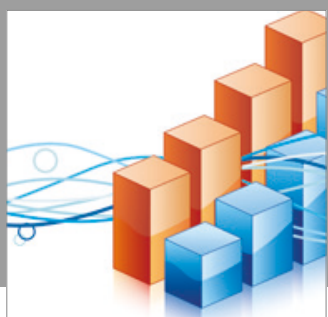

Advances in

Operations Research

vatersals

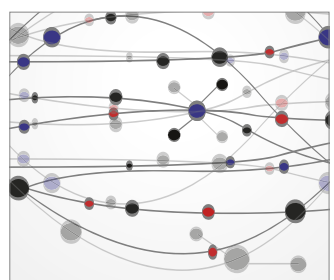

\section{The Scientific} World Journal
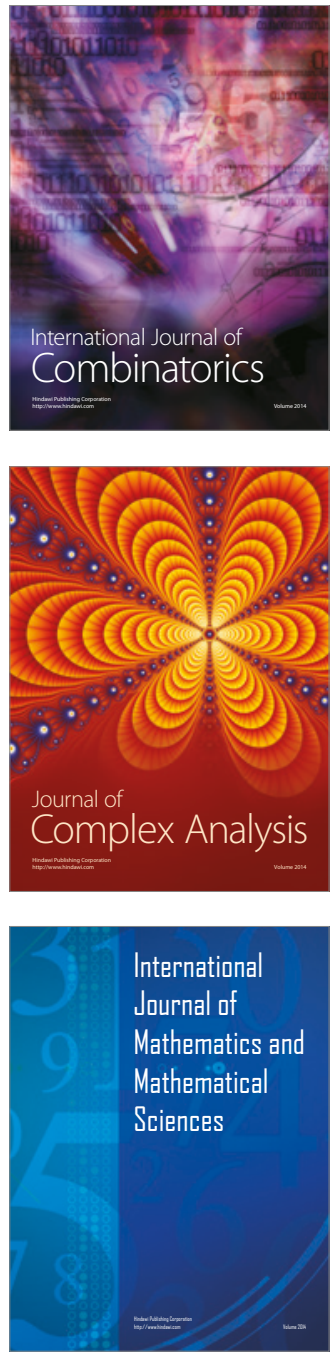
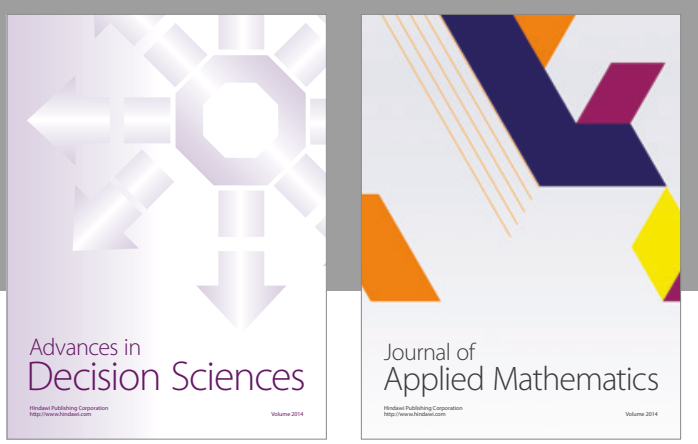

Algebra

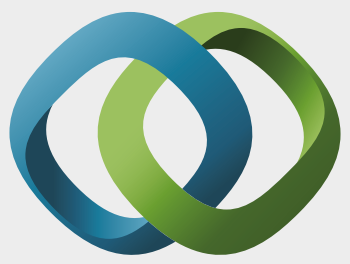

\section{Hindawi}

Submit your manuscripts at

https://www.hindawi.com
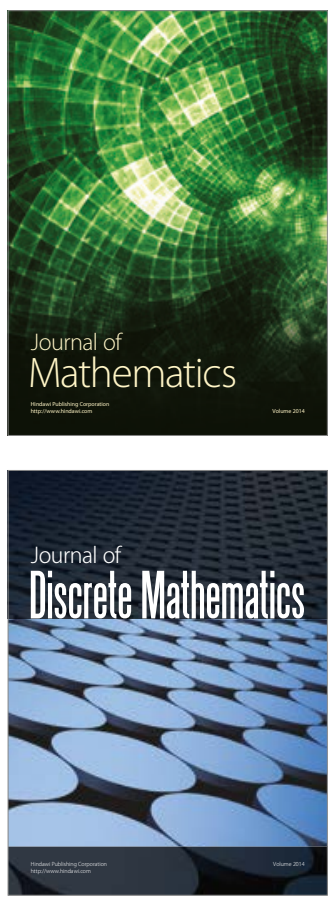

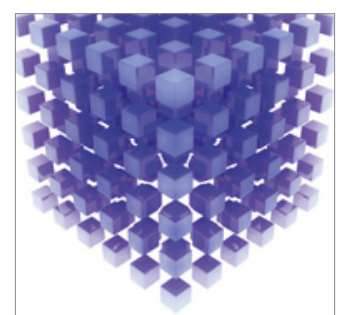

Mathematical Problems in Engineering
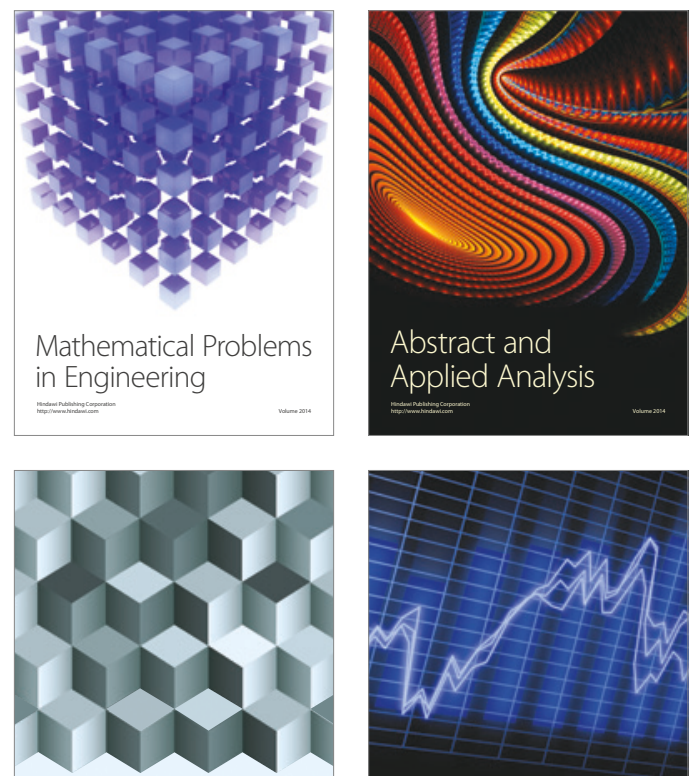

Journal of

Function Spaces

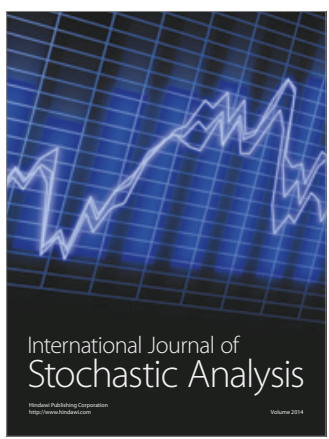

Probability and Statistics
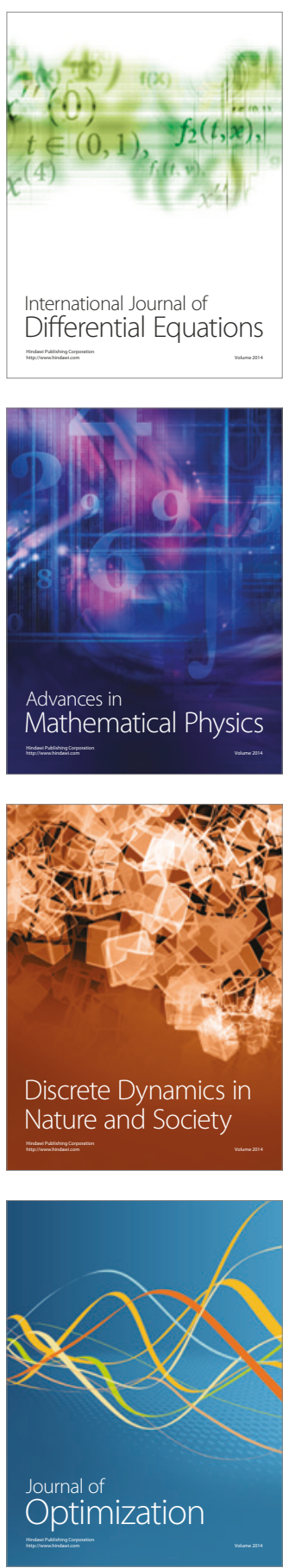